\title{
RELEVANCIA Y APLICABILIDAD EN ÉTICA
}

\author{
«La Ética discursiva no proporciona orientaciones de contenido, \\ sino un procedimiento, pleno de presupuestos, que debe garantizar \\ la imparcialidad en la formación del juicio. El discurso práctico no \\ es un procedimiento para la producción de normas justificadas, sino \\ para probar la validez de normas a título hipotético». \\ p. 132) \\ (J. Habermas: Moralbewu \$tsein und kommunikatives Handeln,
}

1 procedimentalismo de Habermas ha contribuido a la especialización y a avances importantes en la Ética normativa. Ahora bien ¿eso es todo? Las líneas maestras de su rigor en la definición de funciones, el análisis de principios, el cuidado en no traspasar los límites de las cuestiones morales, la defensa del punto de vista moral, la estricta delimitación entre facticidad y validez, etc., proceden del modelo y del lenguaje kantiano. Kant había separado ya las competencias del «experto en Filosofía práctica» de otras posibles competencias, atribuibles al «filósofo práctico». Las respectivas funciones muestran aún hoy por qué la Ética -y no sólo la Ética discursiva- mantiene pretensiones diferentes ante los casos prácticos: el experto sabe lo que se ha de hacer, en qué consisten los fines. En cambio, el filósofo práctico sabe tan sólo de los deberes, ya despojados de lo empírico. Pero ahora las críticas acusan la insuficiencia práctica de esta última modalidad. ¿Cómo resolver los casos? Tal vez, la versión más desvaída de los «filósofos prácticos» ha estado en el origen del resurgir de las éticas especiales, como asunto de expertos, y un tanto al margen del rigor obligado en la Filosofía práctica. ¿Por qué?

En principio, un saber práctico no tiene por qué ser un saber de expertos. Es más, la Filosofía kantiana situaba fuera, en lugar indeterminado, al universo de lo empírico. Ahora bien, ese vacío creado alrededor de la figura del «filósofo práctico», a la manera de los modernos, ha terminado por mostrar ciertos aspectos fallidos de la Ética moderna. Y lo ha hecho, además, en detrimento de las aportaciones positivas de esta figura, el filósofo práctico, que no es ni pretende ocupar el lugar de un experto. Mucho menos el lugar del moralista. Algunas críticas muestran de manera muy acusada las insuficiencias de la Ética, por haber dejado en segundo plano algunas cuestiones prácticas importantes. ¿Qué dificultades son éstas? Nada menos que la escasa aplicabilidad, la ficción de los compromisos universalistas, la idea errada de un sujeto moral sin vínculos con la práctica real, la abstracción convertida en una omisión selectiva, el pesado silencio que cae sobre quienes 
no llegan o no pueden llegar hasta el modelo del agente racional, etc. El modelo liberal reúne un número elevado de tales inconvenientes, pues se ocupa de los agentes morales como ciudadanos y no como individuos concretos; así se ha convertido en el blanco preferente de las objeciones del Comunitarismo, de los republicanos, del Feminismo.

Las páginas siguientes analizan hasta qué punto las deficiencias teóricas de este modelo, formalista y en la línea de la imparcialidad liberal, justifican en realidad la demanda creciente de una «nueva» Ética, ¿qué aportan las éticas especiales a la Filosofía moral? Se trata de: (1) preguntarse si la búsqueda de relevancia supone fragmentar a la Ética en éticas especiales, adoptando la metodología casuística (S. Toulmin, A. Jonsen). (2) El análisis de la «aplicabilidad» en Ética (J. Habermas, K. Günther, R. Alexy) permite extraer algunas conclusiones, a propósito del papel efectivo que desempeñan las éticas aplicadas o especiales. Esto sucede, además, en medio del proceso de avances y críticas, que tanta presencia tienen en los debates de la Filosofía moral contemporánea. Pues las éticas especiales sí han asumido un papel, a veces beligerante, en contra o al margen de las teorías. (3) La diferencia entre knowing how and knowing that (G. Ryle) marca el límite para la «aplicabilidad», entendida en sentido amplio. En principio, no parecía que la expansión de las formas aplicadas de la Ética tuviera una relación directa con las tendencias actuales. Pero sí hay una relación indirecta: la tensión de la Metaética, incluso el esfuerzo empleado en la rehabilitación de la Filosofía práctica, han tenido un cierto contrapeso en las éticas aplicadas. La relevancia práctica tenía que ser recuperada de alguna forma. Pero, ¿había que borrar las diferencias entre un «programa horizontal» y un «programa vertical» en Ética (J. Habermas)?

\section{La búsqueda de relevancia}

M. Sande ${ }^{1}$ se refiere precisamente al fenómeno de cansancio y de descontento difuso, que afecta todavía a la valoración de la teoría ética y política. En su Democracy's Discontent se encuentra el balance de una experiencia colectiva fallida: la Filosofía, situada a distancia del mundo. Algunos opinan que no tuvo ni tendrá éxito una vida que responda a ideales. Sandel se hace eco de este sentimiento generalizado, haber perdido el control sobre la propia existencia, viendo de cerca las dificultades que pesan sobre una Ética de aspiraciones cosmopolitas. En otras versiones la idea es muy similar: el mundo invertido atenaza todavía a la Ética. En ese contexto podemos preguntarnos ¿qué representa el crecimiento de las éticas aplicadas?

\footnotetext{
${ }^{1}$ Sandel, M.: Democracy's Discontent, Harvard University Press, Cambridge, 1996, pp. IX-XI, 1-24.
} 
¿Responden a un esfuerzo por invertir la tendencia «esotérica» de la Filosofía moral como disciplina especializada? ¿Han conseguido situar al universo moral en la contingencia, poner las cosas en su lugar adecuado? Las expectativas que han suscitado las éticas aplicadas son a veces desmedidas. Pesa también su impresionante variedad: Bioética, Ética ecológica, Ética de los negocios, Ética médica, de la educación, la tecnología, las profesiones, de los medios de comunicación, de la actividad política, de los recursos nucleares ${ }^{2} \ldots$

Los Comités de Ética, y la presencia de expertos o asesores en tales materias dentro de las instituciones, muestran el grado de interés que suscitan las éticas especiales ${ }^{3}$. Puede ser un signo favorable. Pero podría tratarse también de algo diferente, de una vía paralela, del todo inerte para la construcción de la teoría filosófica. A veces ofrece una nueva versión de métodos -Nueva Casuística, Nueva Retórica-, que cayeron en desuso hace tiempo por haber estado vinculados a una forma de vida, también desaparecida. Como sucede con algunas formas de cultura y con tradiciones o autoridades religiosas. Las éticas aplicadas ¿son una nueva versión de métodos y prácticas caídas hace tiempo en descrédito? En tal caso ¿cómo podrían contribuir a la revitalización o «rehabilitación» de la Ética como disciplina? La respuesta tiene que ser ambivalente, aun a riesgo de prolongar más de lo debido el capítulo de los preámbulos. Dicho en pocas palabras: las éticas especializadas sí han aportado una nueva relevancia a la Ética pero, también, han creado algunas expectativas distorsionadas sobre la Filosofía práctica y lo que ésta puede decir. La expansión de las éticas aplicadas aspira a tender ciertos puentes allí en donde $\mathrm{Kant}^{4}$ separó ámbitos: la Filosofía pura y, de otro lado, la Filosofía aplicada a la naturaleza humana. Sin embargo, esta nueva relevancia puede ser menos efectiva de lo que aparentaba en un principio. ¿Por qué razones?

$\left(1^{\mathrm{a}}\right)$ La Filosofía práctica de las últimas décadas seguía mostrando un débil interés por los campos y casos de la argumentación práctica. La «rehabilitación» ${ }^{5}$ de la Filosofía práctica marchaba en otra dirección, más constructivista, por así decirlo. Algunos campos argumentales llegaron a ser

\footnotetext{
${ }^{2}$ Puede encontrarse un ejemplo en el trabajo de Lee, S.: «Morality and Nuclear Weapons Policy», Philosophy \& Public Affairs, 19, 1990, pp. 93-106.

${ }^{3}$ Sobre los Comités de Ética y la presencia de los profesionales de las disciplinas de la Filosofía práctica en el tejido institucional, Atienza, M.: «Juridificar la Bioética», Claves de la razón práctica, 61, 1996, pp. 2-15.

${ }^{4}$ Kant, I.: Fundamentación de la Metafisica de las costumbres, Espasa Calpe, Madrid, 1967, p. 55.

${ }^{5}$ La expresión de M. Riedel, Rehabilitierung der praktischen Philosophie, Rombach, Freiburg, 1972.
} 
tan problemáticos como atractivos por su temática; de ahí la necesidad de elaborarlos teóricamente. Sin embargo el resultado, las éticas especiales, a veces ofrecía -y ofrece aúnsoluciones teóricamente deficientes. Por una parte, los campos de la argumentación se convierten en disciplinas independientes, discursos relativamente autónomos, creciendo por el lado de los temas nuevos. Pero permanecen estancadas en cuanto a conceptos y vocabulario moral. Parece que la dedicación a tareas metaéticas se saldó con resultados tanto positivos como totalmente negativos para la Filosofía moral y, ante ese abandono, el método del caso ${ }^{6}$ venía a ofrecer una alternativa: contra la tiranía de los principios, contra la búsqueda infructuosa de un código invariable, contra el sueño imposible de un algoritmo ético. En cuanto al universalismo ¿cómo iba a ganar relevancia práctica para la Ética? ¿Estaba en condiciones de ofrecer una mayor atención hacia el tiempo y el espacio determinados? La casuística se presentaba entonces como una respuesta a las insuficiencias del universalismo.

( $\left.2^{\mathrm{a}}\right)$ El vocabulario y el enfoque casuístico formaron parte, sin embargo, de una metodología anclada aún en etapas precedentes, y en creencias de perfiles reconocibles. En su momento, Pascal había mostrado las muchas deficiencias del procedimiento en el razonamiento moral. Por ello, las importantes ventajas de la prudencia sobre la episteme pueden convertirse también en defectos. ¿Qué puede decir la casuística ahora? Pretende recuperar relevancia, una vez que los principios han mostrado cierta insensibilidad hacia lo particular, e incluso cierta «ceguera moral». S. Toulmin ${ }^{7}$ ha llamado la atención sobre la casuística como método, por su potencialidad para ir más lejos y por ser más flexible que la moralidad de principios universales. Ahora bien, junto a sus ventajas teóricas y prácticas, mantiene vínculos que la mantienen cerca, demasiado cerca de tradiciones y prácticas religiosas, católicas, protestantes, islámicas, rabínicas. El mismo Toulmin propone límites a la «tiranía» de los principios, pero, también, a las diferencias sutiles que aparecen en los casos reales. S. Toulmin y A. Jonsen se refieren a algo así como un «mapa metódico» ${ }^{8}$, que fuese recogiendo semejanzas y diferencias significativas entre los casos. ¿Para qué? Con objeto de resolverlos de forma aceptable y contar con precedentes para los nuevos casos. Pretenden renovar la noción misma de casuística -la Nueva Casuística-, valorando muy favorablemente la contribución realizada hasta la fecha por las nuevas éticas aplicadas. Toulmin concluye: le han «salvado la vida» a la Ética.

\footnotetext{
${ }^{6}$ Jonsen, A. R., Toulmin, S.: The Abuse of Casuistry, University of California Press, Berkeley, 1988, p. VII, p. 7, pp. 304-322.

${ }^{7}$ Toulmin, S.: «The Tyranny of Principles», The Hastings Center Report, 6, 1981 pp. 31-39; «How Medicine Saved the Life of Ethics», en: De Marco, J. P., Fox, R.: New Directions in Ethics, Routledge and Kegan Paul, London, 1986, pp. 265-281.

${ }^{8}$ Jonsen, A.R., Toulmin, S.: The Abuse of Casuistry, p, 14.
} 
$\left(3^{\mathrm{a}}\right)$ M. Singer ${ }^{9}$ ha sido también muy explícito al defender el método de los problemas caso por caso. Es más, se ha mostrado convencido de que la casuística puede hacer de la Ética una ciencia; en razón del procedimiento y de los temas que la conectan con la investigación científica. Sucede así con los temas del medio ambiente. Le parece que la vía del caso por caso ha reabierto la comunicación entre Ciencia y Filosofía; es un hecho que los avances técnicos refuerzan indirectamente una tendencia a la división del trabajo en el interior de la Ética. En muy poco tiempo, han alcanzado un evidente grado de autonomía aquellas disciplinas que se aplican a un ámbito concreto: Bioética, la Ética médica, Ética ecológica, Ética medioambiental, Ética profesional, Ética de los negocios, Ética de la educación. Pero no siempre hay un uso riguroso de la terminología. Por ejemplo, suele haber un uso indistinto de los términos en Bioética y en Ética médica.

Tampoco hay acuerdo sobre los usos del lenguaje, tal vez no demasiado afortunados. Incluso T. Beauchamp ${ }^{10}$, que se ha ocupado con buenos resultados de la Ética médica, se preguntaba sobre si tiene algún sentido separar, como acostumbra a hacerse, Teoría ética y Ética aplicada. Pues no es correcto decir que hay una teoría more geométrico y, en el otro lado, los contextos particulares de la acción. A la vista de las dificultades surgidas, la diversificación de los problemas prácticos parece haber influido en dos sentidos sobre la Filosofía moral: nada o mucho. Por un lado, la expansión de los problemas prácticos ha sido vista con enorme desconfianza, sobre todo desde la Filosofía europea, ya que el crecimiento de la Ética aplicada tiene lugar principalmente en Estados Unidos. Por otro, la presencia de las éticas especiales es un hecho de importancia. Con todo, se trataría de una situación mucho menos novedosa de lo que se pretende. Esto es, la especialización no habría alcanzado cotas suficientes como para cambiar el rumbo de la teoría: no ha dejado sus huellas en la redefinición de los conceptos, ni en una mayor orientación hacia la aplicabilidad, pues no forma parte de la construcción misma de la Ética, de sus aspectos internos. La expansión de los temas prácticos, no se traduce al final en expansión de la Ética. Ni la casuística aparece como la solución que le «salva la vida» a la Ética, como dice Toulmin.

¿Podía realmente «salvarle la vida»? Frente a los pretendidos excesos de la Metaética -y de los filósofos de la moral ${ }^{11}$-, el cambio operado por las

${ }^{9}$ Singer, M.: «Ethics, Science, and Moral Philosophy» en: De Marco, J. P., Fox, R.: New Directions in Ethics, pp. 282-298.

${ }^{10}$ Beauchamp, T. L.: «On Eliminating the Distinction Between Applied Ethics and Ethical Theory», The Monist, 67, 1984, pp. 514-531.

${ }^{11}$ J. Ferrater Mora comentaba la idea de que la Ética no es sólo asunto de los filósofos; Ética aplicada, Alianza, Madrid, 1981, p. 40. 
éticas especiales daba la impresión de haber cerrado el ciclo, en beneficio de un modelo no dependiente de Teoría moral. Pero existe todavía la otra posibilidad: el modelo dependiente. Porque las éticas aplicadas no demuestran resolver los casos prácticos de forma más coherente que un modelo subordinado a la teoría. Por ejemplo, un análisis completo de los casos, dada su complejidad $^{12}$, ha de articularse con vistas a algún principio. Incluso la misma expresión, «éticas aplicadas», puede ser sustituida por otra, más acorde con la dependencia de las éticas especiales son respecto de la teoría: Ética «orientada a la práctica». ¿Es necesario tomar tantas distancias con respecto a la figura del filósofo como experto o como «ingeniero» ${ }^{13}$ ? En algunas ocasiones, preferiríamos que el filósofo práctico fuese también experto en Filosofía práctica, como decía $\mathrm{Kant}^{14}$. Pero la figura del experto es excesiva, extemporánea. Ni la casuística como práctica ni el filósofo como experto pueden asumir funciones, que corresponden al conocimiento y a las competencias de los propios afectados. Ciudadanos competentes, sujetos morales competentes. Es más, el contexto presente sugiere que las desventajas apreciadas en el modelo más teórico, constructivista, liberal, de restricciones y neutralidad práctica, comienzan a parecerse bastante a ventajas importantes. Empezando por la denominada «textura abierta» de los conceptos morales ${ }^{15}$, siguiendo por la «abstinencia epistémica» y llegando hasta la no determinación de las proposiciones evaluativas ${ }^{16}$. Una visión del conjunto permite apreciar ahora que la Moral provisiona $1{ }^{17}$ muestra flexibilidad, mucho mejor que otras soluciones pensadas para «salvar» la relevancia de la Ética. Pues ¿qué hay que salvar en un contexto complejo? La hipótesis de que el knowing how tiene prioridad sobre el knowing that no es nueva, desde luego. Aquí ayuda a justificar por qué las éticas aplicadas son un test importante, pero nada más que un test para la Ética. La Ética sigue siendo el foco central ${ }^{18}$.

${ }^{12} \mathrm{Ch}$. Larmore recuerda que el pluralismo y el desacuerdo entre personas razonables constituye una parte de la experiencia moderna. The Morals of Modernity, Cambridge University Press, Cambridge, 1996, pp. 10-13.

${ }^{13}$ A. Caplan llega a la conclusión de la insuficiencia del modelo del «ingeniero» como cualificación del filósofo para intervenir en los casos prácticos; «Can Applied Ethies Be Effective in Health Care and Shotild ti Strive to Be?», Ethics, 93, 1983, pp. 311-319.

${ }^{14}$ Kant, L: Metafisica de las costumbres, Tecnos, Madrid, 1989, pp. 223-224.

${ }^{15}$ Brennan, J. M.: The Open-Texture of Moral Concepts, MacMillan, London, 1977, p. 94.

${ }^{16}$ Dworkin, R.: «Objectivity and Truth: You'd Better Believe It», Philosophy \& Public Affairs, 1996, pp. 87-139.

${ }^{17}$ Descartes, R.: Discours de la méthode, Garnier-Flammarion, Paris, 1966, p. 51.

18 M. Kettner menciona esta solución; «Bereichspezifische Relevanz. Zur konkreten Allgemeinheit der Diskursethik», en: Apel, K.O0., Kettner, M.: Zur Annwendung der Diskursethik in Politik, Recht und Wissenchaft, Suhrkamp, Frankfurt, 1992, pp. 317-348. 


\section{Aplicabilidad}

Las éticas especiales no forman parte de una etapa post-ética. Por esto hay que tomar en serio la terminología de Kant: principio objetivo y principio subjetivo de la moralidad ${ }^{19}$. Aquél es el principio de la moralidad, pues no depende de las inclinaciones, es puro intellectuali interno. Puro, interno... Despojar al discurso moral de la riqueza y variedad de lo empírico comporta por lo menos tantos riesgos como ventajas. Se traduce, además, en el uso de un tipo de lenguaje, puesto al servicio de la neutralidad, imparcialidad y objetividad del punto de vista moral. Será claro pero árido, en precario equilibrio entre la austeridad y la sequedad; I. Murdoch ${ }^{20}$ señalaba un inconveniente serio de esta elección, más allá de una cuestión de estilo. La transparencia ¿no ofrecerá una traducción empobrecida de la vida moral? El vocabulario moral ¿debería prestar mayor atención a lo que no es cristalino, precisamente porque es real e incompleto? La llamada de atención se repite cada vez con más frecuencia: la Ética de principios y reglas no atiende a la percepción moral, ni a la formación del juicio moral. ¿Por qué relegar nuestra capacidad de percibir las situaciones, las personas, las particularidades ${ }^{21}$ ?

Respuesta: la moralidad de tipo universalista y abstracta no puede contener toda la variedad, particularidad y riqueza de la realidad moral. No puede hacerlo, ¿o no debe? Su aplicabilidad deficiente motiva precisamente la demanda de éticas especiales; como alternativa a un programa general de la Filosofía que, con la época moderna, se aparta de las motivaciones en favor de la racionalidad de las cuestiones prácticas. Es más, el universalismo es un argumento en sí mismo, asociado con frecuencia a las características que definen al punto de vista moral. Punto de vista no comprometido con un contenido determinado ni con intereses reducidos; K. Baier $^{22}$ lo hacía coincidir con el punto de vista de cualquiera. Ser moral es seguir las reglas que van en interés de cualquiera, pese a los sacrificios personales que a veces comportan. M. Singer recuerda, no obstante, el peso de las consecuencias indeseables en el argumento de generalización $^{23}$. Pero sólo el principio de generalización introduce la imparcialidad y la equidad, a fin de que las cuestiones prácticas tengan el mismo tratamiento, para personas similares

\footnotetext{
${ }^{19}$ Kant, L: Gesammelte Schriften, De Gruyter, Berlin, 1979, Bd. XXVII, pp. 1422-1433.

${ }^{20}$ Murdoch, L «Against Dryness; A Polemical Sketch», en: Hauerwas, S., MacIntyre, A.: Revision: Changing Perspectives in Moral Philosophy, University of Notre Dame Press, Notre Dame, 1983, pp. 43-50.

${ }^{21}$ Blum, L.: «Moral Perception and Particularity», Ethics, 101, 1991, pp. 701-725.

${ }^{22}$ Baier, K.: The Moral Point of View, Cornell University Press, Ithaca, 1958, pp. 187-213.

${ }^{23}$ Singer, M. G.: Generalization in Ethics, Atheneum, New York, 197 1, pp. 13-23.
} 
en similares circunstancias. Th. Nagel $^{24}$ identifica así el punto de vista moral con el punto de vista que no está en ningún lugar ni es de nadie en particular, porque trasciende los propios deseos, los elementos irreductiblemente subjetivos del bien. Y J. Habermas introduce el principio de universalización $(\mathrm{U})^{25}$, como regla de argumentación para comprobar la validez de las normas. ¿Con qué efectos?

(1) J. Habermas se refiere de manera expresa a la aplicabilidad como un auténtico problema, reconociendo además que afecta a su propio programa de Ética. El modelo discursivo se ha especializado en una línea bien definida: «Las éticas de tipo kantiano están especializadas en cuestiones de justificación; dejan sin responder cuestiones de aplicación» ${ }^{26}$. Porque ninguna norma contiene las reglas de su aplicación. Porque la Ética discursiva se ocupa preferentemente de asegurar una justificación que sea imparcial. Y porque -como demuestran sus observaciones ${ }^{27}$ a propósito de la teoría reciente de J. Rawls- en la Moral universalista y postconvencional tiene prioridad la validez (incondicionada) sobre consideraciones de bienes y valores (condicionados). Una acepción tan rigurosamente procedimental de Filosofía moral pudiera resultar estrecha e, inclusive, llegaría a dar una falsa idea de la universalidad. Habermas discrepa: haberse marcado como objetivo principal clarificar el punto de vista moral no significa mantener una idea excluyente, demasiado estrecha de universalismo. En fin, el punto de vista moral es ir más allá de los límites, marcados por las comunidades concretas o por valores particulares.

(2) La alternativa entre aplicabilidad y fundamentabilidad sigue presente en los comentarios de K. Günther ${ }^{28}$ sobre la Ética discursiva. La idea general es que no se ha de renunciar a la racionalidad en el ámbito de lo moral y de lo jurídico. Desde el punto de vista del cognitivismo, sin embargo, la aplicación de las normas complica la tarea de su fundamentación; pues la aplicabilidad remite a las situaciones relevantes: éstas tienen que ver sin duda con un orden social, con pautas culturales e incluso con el simple azar. ¿Cómo evitar entonces el rigorismo abstracto por el que se decantan las Éticas

\footnotetext{
${ }^{24}$ Nagel, Th.: The View From Nowhere, Oxford University Press, Oxford, 1986, pp. 8-12.

${ }^{25}$ Habermas, J.: Moralbewu \$tsein und kommunikatives Handeln, Suhrkamp, Frankfurt, 1983, p. 131.

${ }^{26}$ «Moralität und Sittlichkeit», en Kuhlmann, W.: Moralität und Sittlickeit, Suhrkamp, Frankfurt, 1986, p. 27; «On The Cognitive Content of Morality», Proceeding of the Aristotelian Society, 1996, pp. 335-358: Faktizität und Geltung, Suhrkamp. Frankfurt, 1992. pp. 166-237.

${ }^{27}$ «Reconciliation Trought the Public Use of Reason: Remarks On J.Rawls's Political Liberalism», The Journal of Philosophy, 1995, pp. 109-131.

${ }^{28}$ Gunther, K.: Der Sinn der Angemessenheit, Suhrkamp, Frankfurt, 1988, pp. 23-99, 176-215.
} 
universalistas? La versión aristotélica de la moralidad situaba los criterios para juzgar y para aprehender la situación dentro de una forma de vida. En cambio, la versión universalista busca criterios racionales para juzgar, con completa independencia ante las situaciones; luego ¿qué papel desempeñan las situaciones concretas? En principio, ninguno; por lo menos en la versión más rigurosa de la Ética cognitivista. El objetivo principal continuará siendo la fundamentación de normas generales y, en consecuencia, la aplicación imparcial de un procedimiento discursivo no contemplará, no tendrá siquiera por qué indagar por las consecuencias derivadas de tal aplicación. En suma: ninguna aplicabilidad.

(2.1) A la vista de este resultado, bastante previsible, K. Günther propone otro criterio de universalidad, más abierto y, por tanto, con algunas posibilidades en cuanto a la aplicación. Primero, sugiere que es preciso distinguir entre la aplicación del principio de universalidad (U) $\mathrm{y}$, segundo, las situaciones de aplicación. Una versión más débil del principio U será también un principio abierto a la aplicabilidad. Porque la validez de una norma queda asegurada en circunstancias semejantes y para los intereses de cada uno. Por tanto, la norma será aceptable, ya que los intereses de los participantes convierten a aquélla en una norma situada.

(2.2) La aplicación de las normas en las situaciones tiene lugar en el discurso. Por este segundo motivo, las normas pueden ser recontextualizadas, ser reintegradas a los intereses, a tiempos y espacios concretos. Mediante este recurso, K. Günther no anula las diferencias existentes entre la fundamentación y la aplicación, pero sí incorpora para el universalismo tanto la perspectiva social como una mayor sensibilidad hacia el contexto. En respuesta a las críticas al rigorismo de la moral postconvencional, Günther recupera al final un criterio del tipo it depends.

(3) R. Alexy ${ }^{29}$ discrepa de esta solución. Cuestiona incluso el significado de la diferencia entre «fundamentabilidad» $\mathrm{y}$ «aplicabilidad»; ya que todo discurso de aplicación, por lo menos desde la perspectiva de los criterios universalistas ¿no contiene asimismo un discurso de fundamentación? En segundo lugar, es cierto que la «fundamentación» se refiere a la validez normativa, mientras la «aplicación» significa algo distinto, que la norma resulta adecuada para las situaciones concretas. Ahora bien ise trata de discursos esencialmente distintos? Alexy considera, en fin, que el discurso de aplicación no representa lo contrario del otro, el discurso de fundamentación. Tampoco generan formas discursivas independientes entre sí ¿Por qué razón?

29 Alexy, R.: Recht, Vernumft, Diskurs, Suhrkamp, Frankfurt, 1995, pp. 7-8, 52-70; «Zum Begriff des Reschtsprinzips», Rechtstheorie, 1, 1979, pp. 59-87; «Probleme der Diskkurstheorie», Zeitschrift für Philosophische Forschung, 43, 1980, pp. 81-93. 
(3. 1) La fundamentación puede ser de dos tipos: interna y externa. La primera se refiere a la validez de las conclusiones, sin contradicción en las premisas de un argumento y, por tanto, el juicio como consecuencia de enunciados lógicos. La fundamentación externa, en cambio, incluye la aceptabilidad de las premisas, no sólo su corrección. Así, la fundamentación es algo más que consecuencia lógica.

(3.2) En todo caso, la fundamentación se refiere sólo a normas prima facie. Ya que no es posible saber cuáles serán las aplicaciones y a qué situaciones se referirán las normas. Es decir, no puede esperarse que el discurso moral aporte soluciones precisas para una situación determinada. Por eso mismo, no existe tal heterogeneidad entre fundamentar y aplicar las normas.

Aplicar significa dar con la decisión que es correcta para el caso. Pero también remite a una norma. Alexy recuerda que, en todo caso, las situaciones concretas tienen siempre un significado discursivo. Por esta razón, no se oponen a la fundamentación, sino que se convierten en su piedra de toque. En suma, la riqueza y variedad de esas situaciones aporta una perspectiva, mediante la cual el discurso puede corregir, someter a prueba a las normas. Por último, la tesis de Alexy de que, en la interpretación universalista, las normas son normas prima facie -a causa del carácter ideal del discurso-, muestra cierto grado de incertidumbre en las cuestiones prácticas.

\section{Knowing how y knowing that}

La Ética ¿ha de contribuir a dar razón de los sistemas normativos o, por el contrario, al desarrollo de la identidad moral? R. Wiehl ${ }^{30}$ analiza las diferencias entre una Ética racional -desde la pura razón- y una argumentación práctica más compleja, en la cual están también presentes los sentimientos, por ejemplo. La Ética moderna era racional, por lo cual alteró el programa que siguen los agentes morales en su existencia real. Desde otra perspectiva más bien hegeliana, Ch. Taylor ${ }^{31}$ ha llamado también la atención sobre la realidad del multiculturalismo y la correspondiente necesidad de mutuo reconocimiento. La tesis básica de algunos autores dice ahora que el universalismo, el procedimentalismo, el formalismo habrían adoptado la forma más radical y, tal vez, menos relevante, de restricción para la Filosofía práctica actual. ¿Tal vez eran desmesuradas $^{32}$ las expectativas en torno a la actividad filosófica? Entonces, ¿para qué seguir un modelo tan estricto y comprometido con la idea de agente racional?

${ }^{30}$ Wiebl, R.: «Die Vernunft in der menschlichen Unvernunft», en: Metaphysik und Erfahirung, Suhrkamp, Frankfurt, 1996, pp. 277-332.

${ }^{31}$ Taylor, Ch.: «The Polities of Recognition», en: Multiculturalism and «The Politics of Recognition», Princeton University Press, Princeton, 1992, pp. 25-73.

\footnotetext{
${ }^{32}$ Las demandas que dirigen a la Ética han sido analizadas por B. Williams: Ethics and the Limits of Philosophy, Fontana, London, 1985, pp. 23-29.
} 
Probablemente estas restricciones no representan una limitación tan seria como los críticos señalan. Para explicarlo hay que recurrir a la idea de G. Ryle ${ }^{33}$ : knowing how es anterior a knowing that. La inteligencia descubre verdades y las aplica, pero no hace ambas cosas por separado; esto es, sabemos algo porque sabemos hacer algo, conocer es una actividad práctica. Según Ryle, conocer «cómo» y conocer «qué» muestran que el pensamiento afecta al curso de las prácticas y, a la vez, que la inteligencia se ejercita en las prácticas. En el caso de la Ética, este punto de vista significaría que, si conocemos los principios, sabremos también como aplicarlos. Lo cual tiene interesantes consecuencias sobre la anterior separación entre lo teórico y lo aplicado, y entre los programas antagónicos, legados por la Filosofía moderna. Según Ryle, primero descubrimos las reglas, los métodos, no los hechos, ni el qué. Esta hipótesis acortaba las distancias entre la inteligencia teórica y práctica, con cierta ventaja del knowing how sobre el knowing that. Por tanto, conocer es saber hacer una operación o seguir una regla. Ryle añade: un mal razonamiento será también un razonamiento ineficaz. Por lo tanto, conocer «cómo» es una de las condiciones para actuar.

(1) ¿Por qué, entonces, las restricciones de la Filosofía moral? Las restricciones pueden ser de dos tipos, externas e internas. El contexto, lo externo, ha adquirido complejidad, restando así plausibilidad a las explicaciones monocausales de la acción. Las restricciones externas impiden, pues, que la Ética proponga un algoritmo ${ }^{34}$ para guiar acciones en situaciones particulares. Las restricciones internas se traducen, en cambio, en ausencias y silencios cuyo objetivo es preservar un enfoque universalista de tipo kantiano ${ }^{35}$. La Ética discursiva ejemplifica esto último, la prioridad de procedimientos y principios -el knowing how-,pues supone que los agentes son capaces de habla y acción. Son competentes también para tomar sus propias decisiones. Las éticas especiales responden en sentido opuesto, ante las limitaciones externas y a las de tipo interno: la solución de casos reduce la situación de complejidad, reduciendo, al mismo tiempo, la complejidad teórica de la Filosofía moral. Así los objetivos de la Ética procedimentalista y de las éticas aplicadas van en sentido contrario. Al final, la Ética actual y sus distintos programas se asemejan cada vez más a líneas paralelas que no se encuentran en parte alguna.

33 Ryle, G.: «Knowing How and Knowing That. The Presidential Address», Proceedings of the Aristotelian Society, 1945-1946, pp. 1-16.

${ }^{34}$ O’Neill, O.: «Ethical Reasoning and Ideological Pluralism», Ethics, 98, 1988, pp. 705-722. R. Ch. Pielke acentúa la función de los argumentos morales, Critiquing Moral Arguments, University Press of America, New York, 1992, pp. 5-9.

${ }^{35} \mathrm{La}$ alternativa a que se refiere A. Schwartz, «Against Universality», The Journal of Philosophy, 1981, pp. 127-143. 
(2) De modo consecuente, J. Habermas propone que tratemos por separado la perspectiva «horizontal», de relaciones interpersonales, y la perspectiva «vertical» ${ }^{36}$, el proyecto personal de vida. En la línea de Kant, mantiene dos formas de aproximarse a lo moral. Pues ¿cómo puede ser «vertical» o particularista una concepción imparcial de la moralidad? O bien, ¿cómo pensar en un proyecto personal con términos demasiados abstractos? Desde su punto de vista, sólo la justicia aporta un contenido universalista: un bien igual para todos sólo será posible tras haber hecho abstracción de contextos locales y particularistas. El trato separado de lo vertical y horizontal forma parte, en general, de la estrategia propia de una Ética cognitiva. La Ética discursiva lo es, en sentido fuerte incluso; aún al precio de que la justificación moral se asemeje más a las cuestiones teóricas que a los sentimientos morales, a las preferencias reales, a los proyectos de vida: lo vertical. Esta idea de Ética tiene su apoyo más sólido en una teoría de la racionalidad, así como en el supuesto de una relación distanciada, reflexiva, entre la Ética y las formas de vida. La pregunta clave es ¿pueden ser racionales las formas de vida? ${ }^{37}$.

(3) La Ética discursiva no es la única respuesta que ofrece la Filosofía actual. Neoaristotelismo y Utilitarismo comparten el espacio del pensamiento contemporáneo. Sin embargo, aquélla constituye un buen ejemplo para entender tanto la situación de una Ética de principios como, de otro lado, las limitaciones dentro de las cuales se desenvuelve la Filosofía actual. Las ventajas de una Ética cognitivista fuerte se aprecian mejor en el contexto liberal, de pluralismo y de validez para todos los proyectos racionales de vida. Las carencia son, en general, las mismas que se han formulado contra el modelo liberal, y no son escasas. Adoptar un punto de vista liberal implica la prioridad de las libertades: en cuestión de creencias y en cuestión de valores, no puede obligarse a nadie. La cuestión del enforcement estaba presente en las ideas sobre moral, derecho y sociedad, expuestas en su momento por H. L. Hart y P. Devlin ${ }^{38}$. Otros antecedentes teóricos se remontan incluso a las críticas de Hegel, dirigidas contra cualquier interpretación «edificante» de la Filosofía. Ahora bien, la imparcialidad, e incluso las pretensiones de neutralidad y objetividad en modelo liberal, han empezado a mostrar algunos aspectos indeseables. Los afectados hacen notar las malas consecuencias ¿de qué consecuencias se trata? Un número importante de agentes morales encuentran

${ }^{36}$ Habermas, J.: «On the Cognitive Content of Morality», Proceedings of the Aristotelian Society, 1996, pp. 335-358.

37 «Sprechaktheoretische Erläuterungen zum Begriff der kommunikativen Rationalität», Zeitschrift für philosophische Forschung, 50, 1996, pp. 65-91.

${ }^{38}$ Hart, H. L.: «The Legal Enforcement of Morality», en: Law, Liberty, and Morality, Oxford University Press, Oxford, 1978, pp. 1-12; Devlin, P.: «Democracy and Morality» en: The Enforcement of Morals, Oxford University Press, London, 1968, pp. 86-101. 
dificultad para que sus derechos y libertades se conviertan en oportunidades efectivas, para participar en la toma de decisiones.

(4) No se trata entonces de un problema de enforcement, sino de empowerment ${ }^{39}$. De poder, en el sentido de condiciones para desarrollar las capacidades y para participar en la esfera pública. ¿Los beneficiarios? Aquellos que se encuentran con barreras institucionales, quienes están aún lejos de una estructura y distribución equitativas del poder. Pese a que el sistema democrático reconoce formalmente sus derechos. Las críticas al modelo liberal han pasado, pues, del nivel de los principios al nivel de las consecuencias prácticas. S. Benhabib se ha ocupado de algunos aspectos internos del modelo liberal, en tanto C. Sunstein ${ }^{40}$ dirige sus objeciones desde el republicanismo, y hacia la distancia entre la igualdad formal y la igualdad real. Por lo general, los argumentos se centran en la cuestión de la neutralidad liberal: tiene efectos negativos, termina por producir una parcialidad no permisible. Así J. Knight y J. Johnson ${ }^{41}$ se preguntan por las condiciones que perviven en la sociedad liberal, pues ¿hasta qué punto la asimetría puede distorsionar los resultados de la deliberación?

Las observaciones más comunes sobre el liberalismo y sus restricciones, desde los republicanos hasta la crítica feminista, destacan un resultado difícil de asumir y de justificar: la dimensión asimétrica en la relación entre los sujetos. Lo cual significa que la dimensión institucional de la moralidad ha de ser tenida en cuenta. W. Frankena ya advertía que la moralidad es ambas cosas, tanto una cuestión meramente personal como, de otro lado, una institución ${ }^{42}$. ¿Y ahora? Ahora no podemos hacer como si estas críticas no existieran, como si no fuesen relevantes para la justificación teórica y para la actividad práctica. Lo son. ¿De qué manera subsanar las carencias que se han hecho evidentes en las sociedades liberales? ¿Qué tipo de justificación requerirá la Ética que se haga cargo de tales carencias? La justificación, interna o externa ${ }^{43}$, permite avanzar el resultado: cuestiones fácticas junto al

${ }^{39}$ Bachrach, P., Botwinick, A.: Power and Empowerment, University Press, Philadelphia, 1992. pp, 159-172.

${ }^{40}$ Benhabib, S.: «The Methodological Illusions of Modern Political Theory: The Case of Rawls and Habermas», Neue Hefte für Philosophie, 21, 1982, pp. 47-74. Sunstein, C.: «Neutrality in Constitutional Law (with Special Reference to Pornography, Abortion and Surrogacy)», Columbia Law Review, 92, 1992, pp. 1-52; The Partial Constitution, Harvard University Press, Cambridge, 1993.

${ }^{41}$ Knight. J., Johnson, J.: «Aggregation and Deliberation: On the Possibility of Democratic Legitimacy», Political Theory, 22, 1994, pp. 277-296.

42 Frankena, W. K.: «Is Morality a Purely Personal Matter?», Midwest Studies in Philosophy, III, 1978, p. 122-132.

${ }^{43}$ Según la terminología de J. Wroblewski. He comentado este tema en «Ética y tipos de justificación», en: Ética. Procedimientos razonables, Novo Seculo, Iria Flavia, 1994, pp. 43-58. 
nivel de lo contrafáctico. Existe una enorme dificultad para construir un modelo intermedio para la Ética, entre el cognitivismo fuerte y el no cognitivismo, por ejemplo. O entre la argumentación ideal y la argumentación fáctica. ¿Cómo pasar de cuestiones de justificación a cuestiones de participación fáctica? Esto es: subsiste la distancia entre lo vertical y horizontal. Esta conclusión estaba ya implícita en la Ética kantiana.

¿Y las Éticas aplicadas? Las carencias observadas en una Ética de signo cognitivista no parece que tengan su contrapeso más correcto en la independencia de los campos argumentativos: las éticas «aplicadas» pueden ser una insoslayable piedra de toque, pero no una alternativa. Pueden introducir mayor precisión y calidad en los argumentos de la Ética; con lo cual esta opción no alimenta un antagonismo ficticio entre una parte teórica y una parte «aplicada» de la Ética. Tampoco convierte la búsqueda de relevancia en un proceso contra la Filosofía, ni entra en el debate sobre la «tiranía» de los principios. En suma, no pretende compensar la tensión entre el filósofo práctico y el experto. Por una parte, los principios sistematizan la información en cada ámbito, Ciencias de la salud, técnica, medio ambiente, educación, códigos profesionales. Por otra, los campos especiales funcionan como test para la Teoría ética. En la ciencia, las situaciones empíricas permiten situar a las teorías generales con respecto a sus contextos de aplicación. Esto ha de valer también para la Ética, como recuerda $\mathrm{V}$. $\mathrm{Held}^{44}$. Por lo tanto, los casos prácticos o casos difíciles ponen a prueba a los principios mismos, pero sin pretensiones de sustituirlos. ¿El resultado? Las éticas especiales no anulan -no deberíanlas funciones de la teoría. La Ética sigue siendo el foco central. Las éticas especiales no demuestran que haya comenzado una etapa post-ética.

${ }^{44}$ Held, V: «The Political “Testing” of Moral Theories», Midwest Studies in Philosophy, VII, 1982, pp. 343-363. 